\title{
Numerical simulation of bone screw induced pretension: The cases of under-tapping and conical profile
}

Article in Medical Engineering \& Physics · January 2013

DOI: 10.1016/j.medengphy.2013.12.009

CITATION

1

3 authors:

Panagiotis Chatzistergos

Staffordshire University

53 PUBLICATIONS 113 CITATIONS

SEE PROFILE

\section{Stavros Kourkoulis}

National Technical University of Athens

134 PUBLICATIONS 570 CITATIONS

SEE PROFILE
Evangelos A Magnissalis

39 PUBLICATIONS 231 CITATIONS

SEE PROFILE

Some of the authors of this publication are also working on these related projects:

DiaBSmart: Development of a new generation of DIABetic footwear using an integrated approach and SMART materials View project 


\section{NUMERICAL SIMULATION OF BONE SCREW INDUCED PRETENSION:} THE CASES OF UNDER-TAPPING AND CONICAL PROFILE.

$$
\text { Panagiotis E. Chatzistergos }{ }^{(1),(*)} \text {, Evangelos A. Magnissalis }{ }^{(2)} \text { and }
$$

$$
\text { Stavros K. Kourkoulis }{ }^{(3)}
$$

(1) Faculty of Health Sciences, Staffordshire University, Science Centre, Leek Road, ST4 2DF, Stoke on Trent, Staffordshire, UK

(2) BioHexagon LTD, Varnis 36, Nea Smyrni, Attiki, Greece

(3) Unit of Biomechanics, Department of Mechanics, School of Applied Mathematical and Physical Sciences, National Technical University of Athens, Zografou Campus, Theocaris building, 157-73 Attiki, Greece

(*) Corresponding author, tel.: +44 1782295920 e-mail: panagiotis.chatzistergos@staffs.ac.uk 


\section{Abstract:}

Even though screw induced pretension impacts the holding strength of bone screws, its implementation into the numerical simulation of the pullout phenomenon remains a problem with no apparent solution. The present study aims at developing a new methodology to simulate screw induced pretension for the cases of: a) cylindrical screws inserted with under-tapping and b) conical screws. For this purpose pullout was studied experimentally using synthetic bone and then simulated numerically. Synthetic bone failure was simulated using a bilinear cohesive zone material model. Pretension generation was simulated by allowing the screw to expand inside a hole with smaller dimensions or different shape than the screw itself. The finite element models developed here were validated against experimental results and then utilized to investigate the impact of under-tapping and conical angle. The results indicated that pretension can indeed increase a screw's pullout force but only up to a certain degree. Under-tapping increased cylindrical screws' pullout force up to $12 \%, 15 \%$ and $17 \%$ for synthetic bones of density equal to $0.08 \mathrm{~g} / \mathrm{cc}, 0.16 \mathrm{~g} / \mathrm{cc}$ and $0.28 \mathrm{~g} / \mathrm{cc}$ respectively. Inserting a conical screw into a cylindrical hole increased pullout force up to $11 \%$. In any case an optimum level of screw induced pretension exists.

Keywords: Pullout, holding strength, pedicle screw, cohesive material model, synthetic bone, finite element analysis, damage simulation 


\section{Introduction}

Despite the extensive use of pedicle screws and the significant advances in the field of spinal stabilization the possibility of screw loosening and pullout remains and is even higher in the case of osteoporotic patients [1-3].

There are strong indications in the literature that the pretension developed in the vicinity of bone screws during their insertion can significantly influence their pullout strength. Experimental studies performed on synthetic [4] or cadaveric $[5,6]$ bone specimens showed that the holding strength of a cylindrical screw can be improved by under-tapping; namely by inserting the screw into a cylindrical threaded hole which is smaller than the screw itself. Screw insertion with under-tapping causes the core diameter of the threaded hole to expand and the screw hosting material in the vicinity of the screw to compact. In this case the screw's hosting material is compacted uniformly along the length of the screw.

In a previous experimental investigation [4] performed by authors of the present study, it was found that using a tap that is one size smaller than the screw, can increase the pullout force by $9 \%$. Further reduction of the threaded hole dimensions did not result in any statistically significant change of the pullout force.

A combined experimental and numerical analysis of the pullout behaviour of cylindrical self tapping screws was performed by Wu et al. [7]. The authors of this study designed an axisymmetric finite element (FE) model of a screw that is inserted into a threaded hole with dimensions and shape identical to the screw itself. The pretension generated during screw insertion was simulated by introducing a 
temperature change. Even though their model appears to be capable of generating an initial pretension inside the screw's hosting material, the way this capability was utilized is not clear. Numerical results are presented only for cases where the radius of the pilot hole is equal to the screw's core radius.

Moreover, screws with conical core were found to have higher pullout strengths than cylindrical screws with similar thread shape and size [8-13]. Screws with conical core are inserted into cylindrical holes with diameters smaller than the maximum core diameter of the screw. In this case, screw insertion results in a non-uniform compaction of the screw's hosting material. Indeed the screw's hosting material that is closer to the screw's entry site is compacted more than that closer to the screw's tip.

In a previous attempt to simulate the effect of bone compaction in the vicinity of a conical screw that is inserted into a cylindrical hole, the elastic modulus of the screw's hosting material was modified based on an estimate of its volume reduction [8, 9]. The main disadvantage of this approach is that the effect of bone compaction is predefined.

Another interesting approach to the numerical simulation of the pretension that is developed in the vicinity of an implant was presented by Janssen et al. [14] for the case of press-fit acetabular implants. The authors of this study simulated the insertion of the implant as a separate load step.

Considering all the above, the present study aims at developing a reliable and accurate technique to integrate the screw induced pretension to the numerical simulation of the 
pullout phenomenon. The accuracy of the numerical analyses performed here was assessed by comparing numerical and experimental results for four different screw insertion scenarios.

One of the key features for the numerical assessment of a screw's pullout strength is the simulation of the screw's hosting material failure [7, 15-17]. For the purpose of the present study the failure of the screw's hosting material was simulated using a bilinear cohesive zone material model $[15,18]$. The validity of this technique for the simulation of screw pullout has been previously established for cylindrical screws that are inserted into blocks of synthetic bone without any pretension [15]. Its accuracy has also been validated for different densities of synthetic bone [19].

\section{Materials and methods}

\subsection{Experimental study}

Pullout tests were performed with the use of solid rigid polyurethane foam (SRPF) blocks with density equal to $0.16 \mathrm{~g} / \mathrm{cc}$ and material properties similar to osteoporotic cancellous bone (10 pcf SRPF, Sawbones, Worldwide, Pacific Research Laboratories Inc.) and two commercially available pedicle screws, namely Romeo ${ }^{\circledR}$ polyaxial screws for lumbar fixation (Spineart, International Center Cointrin, Genève, Suisse).

The two screws used for the completion of the pullout tests are shown in figure 1. As it can be seen, their thread can be divided into two parts of similar lengths: a cylindrical one and a conical one $\left(\mathrm{L}_{\mathrm{con}}=\mathrm{L}_{\mathrm{cyl}} \approx 20 \mathrm{~mm}\right)$. The main geometrical features of the aforementioned screws are shown in figure 1 while their values are 
presented in table 1. As one can see the two screws have the same pitch $(\mathrm{P})$, the same outer and core radius at their tips $\left(\mathrm{OR}_{\min }, \mathrm{CR}_{\min }\right.$ respectively) and throughout their length they have the same thread depth $(\mathrm{D}=\mathrm{OR}-\mathrm{CR})$ and thread inclination angles $\left(a_{1} / a_{2}\right)$. On the contrary the two screws have significantly different conical angles $\left(\mathrm{a}_{\mathrm{con}}\right)$ and as a result of that they also have different outer and core radius at the transition point from the conical to the cylindrical part of the screw $\left(\mathrm{OR}_{\max }, \mathrm{CR}_{\max }\right.$ respectively). From this point on the pedicle screw with $\mathrm{a}_{\mathrm{con}}=2.5^{\circ}$ and $7.0^{\circ}$ will be referred to as Romeo 2.5 and Romeo 7.0, respectively.

The conical part of the aforementioned screws were inserted into the SRPF blocks through cylindrical holes that were previously prepared using a pillar drill. The insertion depth of the screws was equal to $20 \mathrm{~mm}$. The radius of the cylindrical holes was equal to the minimum core radius of the screws, that is equal to $1.3 \mathrm{~mm}$.

The pullout tests were performed following pertinent international experimental standard (ASTM-F543-02) [20] according to which, the SRPF blocks were fixed to the base of the loading frame (MTS Insight 10kN, MTS Systems Corp., Eden Prairie, $\mathrm{MN}$ ) with the aid of a metallic frame while the screw was suspended from the load cell (MTS 10kN Load Transducer) using a custom-made device (Figure 2). The screw was pulled out of the SRPF block with a constant rate equal to $0.01 \mathrm{~mm} / \mathrm{s}$ while the respective force was measured with a sampling rate of $10 \mathrm{~Hz}$.

Ten tests were performed in total (five tests for each screw) to calculate the mean value and the standard deviation of the pullout force, pullout displacement and the corresponding stiffness for each screw. The results for the two screws were compared 
to each other and their statistical significance was evaluated following one way analysis of variance (ANOVA). The level of statistical significance was considered to be equal to 0.05 .

\subsection{FE modelling and validation}

For the purposes of the present study two different FE models were designed using ANSYS12 software: one FE model for the simulation of under-tapping and cylindrical screw pullout and another one for conical screw pullout. The design of both models was based on the same concept and assumptions.

The pullout phenomenon was simulated with 2D axisymmetric FE models of a bone screw and of its hosting material. The hosting material of the screw was simulated as a homogenous, isotropic, linearly elastic - perfectly plastic material. Its Young's modulus, yield stress and Poisson's ratio were defined according to the values provided by the manufacturer [21] for the SRPF's compressive modulus, compressive strength and Poisson's ratio respectively (table 2). A preliminary numerical analysis revealed that using the values of the tensile modulus and strength instead of the compressive ones does not affect the value of the screw's pullout force.

The experimentally observed failure of the synthetic bone was simulated using a technique previously developed for cylindrical screws that are pulled out of SRPF blocks [15]. According to this technique, the FEs which lay in the vicinity of the screw are connected to each other using bonded contact elements (Conta171, Targe 169) to form a number of successive areas where failure can occur. The areas of possible failure were cylinders in the case of cylindrical screws (Figure 3) and cones 
175 in the case of conical screws. Neighbouring elements at opposite sides of the

176 aforementioned surfaces can break apart from one another, should the tangential stress

177 between them exceeds the shear strength of the SRPF.

179 A bilinear cohesive zone material model was implemented to control mode-II 180 debonding of neighbouring FEs [18]. According to this model, the tangential stress on 181 the interface between a contact pair rises linearly to a critical value (i.e. the shear 182 strength of the SRPF). Beyond this point, any further increase of the relative sliding 183 causes a non-reversible decrease of the tangential stress leading to the complete 184 debonding of the contact pair. From this point on the interface conditions between the initially bonded pair of elements change to simple contact with friction.

As far as the macroscopic behaviour of the model is concerned, debonding of neighbouring elements reduces the total force that resists pullout and causes a clear 189 drop of the force in the force/displacement graph. Indeed the force in the numerically calculated force/displacement graph reaches a maximum value and then drops with increasing displacement. The maximum value of the force is stored as the screw's pullout force. Even though the simulation continues beyond the point where the value of the force starts dropping the solution process becomes slower and finally it stops

194 due to non-convergence. In any case the ultimate force, namely the force calculated 195 for the last sub-step of the solution where convergence was achieved, is always lower than the pullout force.

Taking under consideration the magnitude difference between the Young 's modulus 199 of the screw and of its hosting material, the screw was considered to be rigid [19]. 
Moreover the FE model of the bone screw was designed in a way that enabled the modification of its dimensions and shape. The initial geometry of the screw's FE model was modified to fit inside a cylindrical threaded hole, with dimensions similar to the holes drilled for the pullout testing. In the case of cylindrical screws inserted with under-tapping, the initial core diameter of the screw was modified and set equal to the core diameter of the threaded hole (Figure 4). In the case of conical screws the initial value of the big core diameter of the screw (which is the core diameter of the cylindrical part of the screw) was set equal to the threaded hole's core diameter (Figure 4).

The simulation was performed in two steps to incorporate the effect of pretension development into the simulation of the pullout phenomenon. During the first step the radii of the FE model of the screw were extended to reach their actual values, while during the second simulation step the screw was pulled out from its hosting material (Figure 4). More specifically during the second load step a displacement was imposed to the screw in the pullout direction with the help of a pilot-node. The value of the imposed displacement was $0.5 \times$ screw's insertion depth. This relatively high displacement value was used to ensure the failure of the screw's hosting material. Indeed the exact value of the imposed displacement has no effect on the calculation of the pullout force. The solution process stops when it reaches a point of nonconvergence.

The accuracy of the numerical analysis was assessed by comparing the numerical results with corresponding experimental ones. For that purpose the FE model of the 
pullout phenomenon was modified to closely match the geometry, size and insertion depth of the screws that were used for the experiments.

In the case of cylindrical screws and under-tapping the accuracy of the numerical analysis for was assessed by comparing the numerical results with corresponding experimental ones from a previous investigation performed by authors of this study [4]. Experimental data for two different conditions of under-tapping were used. In the context of that study a cylindrical pedicle screw with core and outer radius equal to $2.75 \mathrm{~mm}$ and $3.75 \mathrm{~mm}$ respectively (CD Horizon Legacy MAS(Ti) $7.5 \times 50 \mathrm{~mm}$, Medtronic Sofamor Danek, Memphis,TN), was pulled out from blocks of synthetic bone with material properties similar to osteoporotic bone (10 pcf SRPF, Sawbones, Worldwide, Pacific Research Laboratories Inc.). Screw insertion was performed with under-tapping. Two different under-tapping ratios were tested, namely the ratio of the screw's core radius divided by the respective radius of the threaded hole $\left(\mathrm{CR}^{\text {Screw }}\right.$ $\left./ \mathrm{CR}^{\mathrm{TH}}\right)$. More specifically the screw was inserted into threaded holes that were smaller than the screw itself by $0.5 \mathrm{~mm}$ and $1.0 \mathrm{~mm}$ (under-tapping ratios equal to 1.2 and 1.6 respectively). The case where the threaded hole had identical size and shape to the screw was also tested (under-tapping ratio 1.0).

In the case of conical screws, the accuracy of the FE analysis was assessed based on the pullout tests performed in the context of this study for the Romeo 2.5 and Romeo 7.0 screws. The experimental force vs. displacement curves and the experimental pullout forces were compared with the respective numerical ones. 
After its validation the FE model of cylindrical screw's pullout was utilized to investigate the impact of under-tapping on pullout strength. A cylindrical screw with core and outer radius equal to $2.75 \mathrm{~mm}$ and $3.75 \mathrm{~mm}$ was simulated inside a cylindrical threaded hole of similar size and shape and its pullout force was calculated

255 for different under-tapping ratios (i.e. $\mathrm{CR}^{\mathrm{Screw}} / \mathrm{CR}^{\mathrm{TH}}$ ). Starting from an under-tapping ratio of 1 (no under-tapping) the threaded hole's core radius was decreased with increments of $0.1 \mathrm{~mm}$. The parametric investigation was terminated when the results 258 indicated that any further increase of the under-tapping ratio will have no effect to the 259 value of the pullout force. This procedure was repeated for three different SRPF densities: $0.08 \mathrm{~g} / \mathrm{cc}, 0.16 \mathrm{~g} / \mathrm{cc}$ and $0.24 \mathrm{~g} / \mathrm{cc}$. The material properties of these SRPFs were defined according to literature [21] and their values are shown in table 2.

The FE model of conical screw's pullout was utilized to investigate the impact of conical angle and pretension to pullout strength. The minimum core and minimum outer radius of the screw were kept constant while the respective maximum radii were modified to produce conical threads with different conical angles. Eight different values of the screw's conical angle ranging from $0^{\circ}$ to $7^{\circ}$ were simulated. Two different

268 simulations were performed for each one of these conical angles, to quantify the impact of pretension to a conical screw's holding strength. The pretension generated

270 inside the synthetic bone during screw insertion was taken under consideration during

271 the first simulation but excluded from the second one. During the first simulation, 272 pretension generation was simulated by inserting the conical screw inside a 273 cylindrical threaded hole, with core and outer radius similar to the minimum core and 
outer radius of the screw. On the contrary during the second simulation the conical screws were simulated inside conical threaded holes with size and shape identical to the screw itself. This way no pretension was developed inside the screw's hosting material. The material properties of the screw's hosting material were those of a synthetic bone simulating osteoporotic cancellous bone (10 pcf SRPF, Sawbones, Worldwide, Pacific Research Laboratories Inc.).

\section{Results}

\subsection{Experimental study}

All specimens exhibited similar mechanical behaviour and failed under shear. The failure appears on an almost conical surface which connects the edges of the threads of the screw. As it can be seen in figure 5, the failure surface in the case of the Romeo 7.0 screw can be described as a cone with conical angle $\approx 7^{\circ}$, the same as the screw itself. The material between the surface of failure and the surface of the screw is extracted from the block together with the screw. Representative force vs. displacement curves of pullout test are shown in figure 6 . The peak value of the force is the screw's pullout force, while the displacement corresponding to this force is the pullout displacement. The tangent of the angle between the linear part of the curve and the $\mathrm{X}$ axis corresponds to the stiffness of the screw - screw's hosting material complex. The results of the pullout test are shown in detail in table 3 .

One way ANOVA indicated that Romeo 7.0 screw has statistically significant $(\mathrm{P}<$ 0.05) higher pullout force than the Romeo 2.5 screw, while there are no statistically significant differences in terms of pullout displacement and stiffness $(\mathrm{P}>0.05)$. 
3.2 FE modelling and validation

301 Figures 6 and 7 depict a clear overview of the simulation process. In the case of 302 cylindrical screws with under-tapping (Figure 6) the screw's radii expand during the 303 first load step, generating a strong and relatively uniform stress field in the vicinity of 304 the screw. During the second load step, the screw is pulled out of its hosting material until failure of the synthetic bone. Debonding is observed in the vicinity of the deepest thread (namely the most distant one from the free surface). Debonding of neighbouring elements generates a sudden stress relief in this region and a discontinuity of the stress field. The strong stress concentration at the edges of the SRPF's free surface are caused by the model's supports (figure 3).

In the case of conical screw's pullout (Figure 7) the shape of the screw is gradually changed from cylindrical to conical to simulate the generation of pretension around the screw. In this case, the stress field developed in the vicinity of the screw is more severe near the free surface of the SRPF block as expected. During the second load step, the screw is pulled out from its hosting material. Most of the pullout load appears to be carried by the thread closest to the free surface.

In terms of validation. In the case of under-tapping the experimental/ numerical pullout forces for under-tapping ratios equal to $1.0,1.2$ and 1.6 were $438 \mathrm{~N} \pm 5 \mathrm{~N} /$ $440 \mathrm{~N}, 480 \mathrm{~N} \pm 7 \mathrm{~N} / 506 \mathrm{~N}$ and $481 \mathrm{~N} \pm 9 \mathrm{~N} / 505 \mathrm{~N}$ respectively. As it can be seen the difference between the numerical simulations and the experiments [4] is less that

$3225 \%$ for all three cases. Moreover the FE models were able to simulate the 323 macroscopic response of the SRPF block - bone screw complex to loading. 
Representative numerical and experimental results are shown in figure $6 \mathrm{~A}$ for the case of under-tapping ratio equal to 1.2. As it can be seen the numerically calculated force vs. displacement curve appears to be in good agreement with the experimental one.

The numerically calculated pullout forces for the Romeo 2.5 and Romeo 7.0 screws were equal to $326 \mathrm{~N}$ and $381 \mathrm{~N}$ respectively. Comparing these values with the respective experimental ones (table 3) gives a difference that is lower than $3 \%$. Moreover, the FE models were able to simulate with satisfactory accuracy the overall response of the SRPF block - bone screw complex (Figure 6B).

\subsection{Parametric analyses}

The pullout force calculated for different densities of the synthetic bone and different under-tapping ratios can be seen in figure 9. In the case of the synthetic bone with density equal to $0.08 \mathrm{~g} / \mathrm{cc}$, pullout force increased significantly with under-tapping ratios ranging from 1 to 1.12 . Indeed the pullout force calculated for under-tapping ratio equal to 1 was $126 \mathrm{~N}$, while for under-tapping ratio equal to 1.12 the pullout force was $143 \mathrm{~N}$, equivalent to $12 \%$ increase. Increasing the under-tapping ratio to values greater than 1.12, did not affect the value of the pullout force. The pullout force calculated for under-tapping ratio equal to 1.17 was again $143 \mathrm{~N}$.

In the case of SRPFs with density equal to $0.16 \mathrm{~g} / \mathrm{cc}$ and $0.28 \mathrm{~g} / \mathrm{cc}$, pullout force increased significantly with under-tapping ratio for ratios up to 1.17 (Figure 9). The pullout force for these two synthetic bones was $440 \mathrm{~N}$ and $894 \mathrm{~N}$ for under-taping 
ratio equal to 1 , while for under-tapping ratio equal to 1.17 pullout force was $507 \mathrm{~N}$ and $1048 \mathrm{~N}$ respectively, equivalent to $15 \%$ and $17 \%$ increase respectively. Increasing the under-tapping ratio to 1.22 had no significant effect to the values of the pullout force (Figure 9). The pullout force calculated for synthetic bones of densities equal to $0.16 \mathrm{~g} / \mathrm{cc}$ and $0.28 \mathrm{~g} / \mathrm{cc}$ and under-tapping ratio equal to 1.22 was $506 \mathrm{~N}$ and $1050 \mathrm{~N}$ respectively.

The pullout force calculated for different values of the conical angle can be seen in figure $10 \mathrm{~A}$, for the cases where initial pretension is either included or excluded from the numerical simulation. As it can be noticed, pullout force increases linearly with conical angle when no pretension phenomena are included to the analysis. On the contrary when pretension is included to the numerical simulation the increase is nonlinear. The pullout forces calculated in this case are higher than the respective ones calculated without any pretension. Comparing the results for these two series of simulations indicates that pretension has a positive effect on screw pullout strength (Figure 10B). The maximum impact of pretension was calculated for a conical angle equal to $2^{\circ}$. In this case pretension improved the screw's pullout strength by $11.4 \%$. The benefit of pretension on pullout strength becomes weaker for conical angles greater than $2^{\circ}$. Indeed for conical angle equal to $7^{\circ}$ pretension causes only a $0.4 \%$ increase of the pullout force. 


\section{Discussion and conclusions}

375

It is well established in literature that the stress developed around a bone screw during insertion can significantly influence its stability [4-6, 8-13, 22]. More specifically, it is indicated that the fixation strength of a bone screw can be improved by under-tapping [4-6] or the use of screws with conical core [8-13]. In the case of under-tapping a cylindrical screw is inserted into a previously opened threaded hole which is smaller than the screw itself. In this case the core of the screw compresses and compacts the bone which lies in its vicinity in the radial direction. The core diameter of the threaded hole is expanded until it becomes equal to the diameter of the screw core. The strong contact pressure that is generated on the interface between the cores of the screw and the threaded hole, also generate frictional forces which resist screw's rotation. If the screw is a self-tapping one then its threads will cut their way through the hosting material of the screw. In this case the pressure field developed on the surface of the threads would be relatively weak.

On the other hand, screws with conical core are inserted into bone using previously opened cylindrical holes. In this case screw insertion causes the core of the hole to change size and shape. The shape of the hole changes from cylindrical to conical causing the screw's hosting material near the screw's insertion site to be compressed more than the material near the screw's tip.

Despite its significance, the pretension that is developed during screw insertion has not yet been fully incorporated into FE simulations of screw pullout. Hsu, Chao et al. $[8,9]$ were the first who tried to simulate the impact of inserting a conical screw into a 
cylindrical hole to the screws pullout strength. Their simulation was based on the idea that the material properties of a screw's hosting material are altered in the vicinity of the screw as a result of material compaction. The main limitations of this approach is the fact that the effect of bone compaction is predefined and that the actual pullout force is not calculated.

Indeed, assuming that the elastic modulus of the screw's hosting material is a function of volume reduction means that screw fixation strength will continuously increase with compaction. On the other hand there is strong evidence in the literature that fixation strength increases with compaction only up to a certain level [4, 23, 24].

The impact of the relative dimensions of screw and hole to the screw's pullout strength was investigated experimentally by authors of the present study [4]. A cylindrical pedicle screw was inserted into blocks of synthetic bone using threaded and cylindrical holes of different sizes and the pullout force was measured. The results indicated that there is an optimum ratio of the threaded or cylindrical hole's radius over the respective screw's radius.

The FE model of Hsu, Chao et al. [8, 9] was capable of calculating the reaction force for a small value of imposed displacement. The value of the reaction force for a constant value of pullout displacement is directly correlated to the stiffness of the screw - hosting material complex rather to the screw's pullout force. According to literature $[11,25-30]$ and also according to the respective international experimental standard [20] the most appropriate way to quantify the fixation strength of a bone screw is to measure its pullout force. 
425 In this context the present study's aim was to establish a new method for simulating

426 the pretension that is developed inside the hosting material of a screw during screw

427 insertion and to incorporate it in the numerical simulation of the pullout phenomenon.

428 The screw was simulated inside a cylindrical threaded hole with dimensions similar to

429 the holes drilled for pullout testing. The initial dimensions of the screw's FE model

430 were modified to match the dimensions of the cylindrical threaded hole. The

431 simulation was performed in two steps: 1) Pretension generation, 2) pullout. Preten-

432 sion generation was simulated by extending the screw's radii to reach their actual

433 values. After the completion of this load step a displacement at the pullout direction

434 was imposed to the screw until the failure of its hosting material.

435

436

444 For cylindrical screws that are inserted with under-tapping into blocks of synthetic

The experimentally observed failure of the synthetic bone was simulated by implementing a bilinear cohesive material model [18]. This methodology has been proven to give reliable estimations of the pullout force of cylindrical screws that are inserted into blocks of synthetic bone through cylindrical threaded holes of identical size and shape to the screw itself [15]. The main limitation of this approach for the simulation of synthetic bone's failure is that the geometrical domain where failure can occur has to be known in advance [16].

bone it has been proven previously that failure occurs on a cylindrical surface that includes the screw $[4,15]$. Moreover for the case of conical screws it was proven here that failure occurs on a conical surface which includes the screw. These experimental observations indicate that in both cases the screw's hosting material fails under shear 
and that both are eligible for using a cohesive material model to simulate synthetic bone failure.

The accuracy of this FE model was validated by comparing the numerically calculated force vs. displacement curves and also the values of the pullout force with experimental ones for two different cases of under-tapping ratios and for two different conical screws. This comparison indicated that the proposed methodology for introducing pretension in the simulation of conical screw pullout enables the accurate assessment of its pullout strength.

After validation two different parametric analyses were performed. The results of the first parametric study demonstrated that under tapping can significantly increase the pullout force of bone screws. Indeed the impact of under tapping is more pronounced in the case of denser and "stronger" synthetic bones. More specifically under tapping was able to increase pullout force up to $12 \%, 15 \%$ and $17 \%$ in the cases of synthetic bones with density equal to $0.08 \mathrm{~g} / \mathrm{cc}, 0.16 \mathrm{~g} / \mathrm{cc}$ and $0.28 \mathrm{~g} / \mathrm{cc}$ respectively. Moreover an optimum value of the under tapping ratio exists which appears to be influenced by the mechanical properties of the screw's hosting material. Increasing the under tapping ratio beyond this value has no significant effect on the screw's pullout force.

In the context of the second parametric analysis the minimum core and outer radii of a conical screw were kept constant while the maximum ones were modified to produce different conical angles. According to literature the outer radius of a screw is one of the most important parameters for its fixation strength. Indeed a screw's pullout force increases linearly with its outer radius [15, 31-33]. For the purpose of this study the 
impact of pretension was quantified separately from that of the maximum outer radius.

The results of this parametric analysis indicated that using a bone screw with a conical core generates a pretension inside the screw's hosting material which can improve the screw's fixation strength. The impact of the pretension to the screw's fixation strength appears to be stronger for relatively small conical angles. Indeed for a conical angle equal to $2^{\circ}, 11.4 \%$ of the screw's pullout force is attributed to the pretension generated during screw insertion. On the other hand in the case of a screw with conical angle equal to $7^{\circ}$ only a $0.4 \%$ of its pullout force is a result of pretension.

Based on the above it could be deduced that in the case of conical screws with big conical angles the best way to improve their short-term fixation strength would be to insert them into conical holes instead of cylindrical ones. The conical angles of these holes should be a couple of degrees smaller than the screw's itself. A possible way to open a conical hole that is smaller than the screw could be to use another screw of similar shape but smaller in size. In a previous experimental investigation performed by authors of the present study it was concluded that using a self-tapping screw to prepare a threaded hole can be as efficient as if a tap was used [4].

The main limitation of the present study stems from the fact that synthetic bone cannot simulate all aspects of the mechanical behaviour of cancelous bone. Nevertheless synthetic bone can be used to perform comparative analyses and draw useful and clinically relevant conclusions [34]. One of the important aspects of bone tissue that cannot be simulated using synthetic bone is the tissues adaptive response to 
499 loading. For this reason the results of this study in terms of fixation strength

501

502

503

504

505

506

507

508

509

510

511

512

513

514

515

516

517 correspond to the first post-operative weeks.

At this point is should be stressed out that the accuracy of the novel technique proposed here for the simulation of pretension has been validated only for the case of a synthetic bone with density equal to $0.16 \mathrm{~g} / \mathrm{cc}$. As a result of that interpreting the numerical results for surrogate bones of different densities should be done with caution. Indeed the FE analyses can predict the maximum possible pretension-induced improvement of a screw's pullout strength. The main prerequisite for this prediction to be accurate is that the screw's hosting material fails during pullout under shear and not during screw insertion. Unfortunately under-tapping also carries the risk of a catastrophic failure during screw insertion [35]. Moreover it is possible that in the case of SRPFs that are denser than $0.16 \mathrm{~g} / \mathrm{cc}$ an 'under-tapping limit' exists beyond which the integrity of the screw's hosting material is jeopardised during screw insertion. The simulation of this type of failure was beyond the scope of the present study.

According to literature under-tapping can lead to pedicle wall breach [35]. Investigating numerically the possibility of pedicle wall breach requires an accurate simulation of the three-dimensional (3D) geometry of the vertebra and of the screw. Investigating the impact of vertebral $3 \mathrm{D}$ geometry was beyond the scope of the present study and therefore the geometry of the screw and of its hosting material was simplified. 
523 On the other hand the main contribution of the present study is the implementation of

524 a novel method for incorporating the impact of different screw insertion techniques

525 and pretension to the FE simulation of the pullout phenomenon.

526

527

528

529

530

531

532

533

534

535

536

537

538

539

540

541

542

543

544

545

546

547 
References:

549

550

[1] Okuda S, Miyauchi A, Oda T, Haku T, Yamamoto T, Iwasaki M. Surgical

$\underline{551}$

complications of posterior lumbar interbody fusion with total facetectomy in 251

552

patients. J Neurosurg Spine. 2006;4:304-9.

553

[2] Esses SI, Sachs BL, Dreyzin V. Complications associated with the technique of pedicle screw fixation. A selected survey of ABS members. Spine (Phila Pa 1976).

555 1993;18:2231-8; discussion 8-9.

$\underline{556}$

[3] Ponnusamy KE, Iyer S, Gupta G, Khanna AJ. Instrumentation of the osteoporotic spine: biomechanical and clinical considerations. Spine J. 2011;11:54-63.

558 [4] Chatzistergos PE, Sapkas G, Kourkoulis SK. The Influence of the Insertion

559 Technique on the Pullout Force of Pedicle Screws An Experimental Study. Spine.

$560 \quad$ 2010;35:E332-E7.

561 [5] Halvorson TL, Kelley LA, Thomas KA, Whitecloud TS, 3rd, Cook SD. Effects of

562 bone mineral density on pedicle screw fixation. Spine (Phila Pa 1976). 1994;19:2415-

56320.

564 [6] Kuklo TR, Lehman RA, Jr. Effect of various tapping diameters on insertion of

565 thoracic pedicle screws: a biomechanical analysis. Spine (Phila Pa 1976).

$566 \quad 2003 ; 28: 2066-71$.

567 [7] Wu Z, Nassar SA, Yang X. Pullout performance of self-tapping medical screws. J

568 Biomech Eng. 2011;133:111002.

569 [8] Hsu CC, Chao CK, Wang JL, Hou SM, Tsai YT, Lin J. Increase of pullout

570 strength of spinal pedicle screws with conical core: biomechanical tests and finite

571 element analyses. J Orthop Res. 2005;23:788-94. 

strength in conical pedicle screws: biomechanical tests and finite element analyses. J

574 Spinal Disord Tech. 2008;21:130-8.

575 [10] Abshire BB, McLain RF, Valdevit A, Kambic HE. Characteristics of pullout

576 failure in conical and cylindrical pedicle screws after full insertion and back-out.

577 Spine J. 2001;1:408-14.

578 [11] Kwok AW, Finkelstein JA, Woodside T, Hearn TC, Hu RW. Insertional torque 579 and pull-out strengths of conical and cylindrical pedicle screws in cadaveric bone. 580 Spine (Phila Pa 1976). 1996;21:2429-34.

581 [12] Kim YY, Choi WS, Rhyu KW. Assessment of pedicle screw pullout strength based on various screw designs and bone densities-an ex vivo biomechanical study.

583 Spine J. 2012;12:164-8.

584

585

[13] Krenn MH, Piotrowski WP, Penzkofer R, Augat P. Influence of thread design on pedicle screw fixation. Laboratory investigation. J Neurosurg Spine. 2008;9:90-5.

[14] Janssen D, Zwartele RE, Doets HC, Verdonschot N. Computational assessment of press-fit acetabular implant fixation: the effect of implant design, interference fit, bone quality, and frictional properties. Proc IMechE 2009;224:67-75.

[15] Chatzistergos PE, Magnissalis EA, Kourkoulis SK. A parametric study of cylindrical pedicle screw design implications on the pullout performance using an experimentally validated finite-element model. Med Eng Phys. 2010;32:145-54.

[16] Feerick EM, McGarry JP. Cortical bone failure mechanisms during screw pullout. J Biomech. 2012;45:1666-72.

[17] Feerick EM, Liu XC, McGarry P. Anisotropic mode-dependent damage of cortical bone using the extended finite element method (XFEM). J Mech Behav Biomed Mater. 2013;20:77-89. 
597

598

599

600

601

602

603

604

605

606

607

608

609

610

611

612

613

614

615

616

617

618

619

620

[18] Alfano G, Crisfield MA. Finite element interface models for the delamination analysis of laminated composites: Mechanical and computational issues. International Journal for Numerical Methods in Engineering. 2001;50:1701-36.

[19] Chatzistergos PE, Spyrou CE, Magnissalis EA, Kourkoulis SK. Dependence of the pullout behaviour of pedicle screws on the screw - hosting material relative deformability. International Journal of Computer Aided Engineering and Technology. 2013;5:343-61.

[20] ASTM. F 543 - 02 Standard Specification and Test Methods for Metallic Medical Bone Screws. Medical Device Standards and Implant Standards2004.

[21] Sawbones.2013.Sawbones webpage,http://www.sawbones.com/.

[22] Silva P, Rosa RC, Shimano AC, Defino HL. Effect of pilot hole on

biomechanical and in vivo pedicle screw-bone interface. Eur Spine J. 2013.

[23] Steeves M, Stone C, Mogaard J, Byrne S. How pilot-hole size affects bone-screw pullout strength in human cadaveric cancellous bone. Can J Surg. 2005;48:207-12. [24] Ansell RH, Scales JT. A study of some factors which affect the strength of screws and their insertion and holding power in bone. J Biomech. 1968;1:279-302. [25] Hitchon PW, Brenton MD, Coppes JK, From AM, Torner JC. Factors affecting the pullout strength of self-drilling and self-tapping anterior cervical screws. Spine (Phila Pa 1976). 2003;28:9-13.

[26] Leggon R, Lindsey RW, Doherty BJ, Alexander J, Noble P. The holding strength of cannulated screws compared with solid core screws in cortical and cancellous bone. J Orthop Trauma. 1993;7:450-7.

[27] Lyon WF, Cochran JR, Smith L. Actual Holding Power of Various Screws in Bone. Ann Surg. 1941;114:376-84. 
621 [28] Pfeiffer FM, Abernathie DL, Smith DE. A comparison of pullout strength for

622 pedicle screws of different designs: a study using tapped and untapped pilot holes.

623 Spine (Phila Pa 1976). 2006;31:E867-70.

624 [29] Reitman CA, Nguyen L, Fogel GR. Biomechanical evaluation of relationship of

625 screw pullout strength, insertional torque, and bone mineral density in the cervical

626 spine. J Spinal Disord Tech. 2004;17:306-11.

627 [30] Wittenberg RH, Lee KS, Shea M, White AA, 3rd, Hayes WC. Effect of screw

628 diameter, insertion technique, and bone cement augmentation of pedicular screw

629 fixation strength. Clin Orthop Relat Res. 1993:278-87.

630 [31] Asnis SE, Ernberg JJ, Bostrom MP, Wright TM, Harrington RM, Tencer A, et al.

631 Cancellous bone screw thread design and holding power. J Orthop Trauma.

$632 \quad 1996 ; 10: 462-9$.

633 [32] DeCoster TA, Heetderks DB, Downey DJ, Ferries JS, Jones W. Optimizing bone

634 screw pullout force. J Orthop Trauma. 1990;4:169-74.

635 [33] Gausepohl T, Mohring R, Pennig D, Koebke J. Fine thread versus coarse thread.

636 A comparison of the maximum holding power. Injury. 2001;32 Suppl 4:SD1-7.

637 [34] Schoenfeld AJ, Battula S, Sahai V, Vrabec GA, Corman S, Burton L, et al.

638 Pullout strength and load to failure properties of self-tapping cortical screws in

639 synthetic and cadaveric environments representative of healthy and osteoporotic bone.

$640 \quad$ J Trauma. 2008;64:1302-7.

641 [35] Chin KR, Gibson B. The risks of pedicle wall breech with larger screws after

642 undertapping. Spine J. 2007;7:570-4.

643

644 
Tables:

646

647 Table 1: The geometrical quantities that describe the geometry of the threads of

648 Romeo 2.5 and Romeo 7.0 screw.

\begin{tabular}{|c|c|c|c|c|c|c|c|c|}
\hline Screw & $\begin{array}{c}\mathbf{P} \\
(\mathbf{m m})\end{array}$ & $\begin{array}{c}\mathbf{D} \\
(\mathbf{m m})\end{array}$ & $\begin{array}{l}\mathbf{O R}_{\min } \\
(\mathbf{m m})\end{array}$ & $\begin{array}{l}\mathbf{C R}_{\text {min }} \\
(\mathbf{m m})\end{array}$ & $\begin{array}{c}\mathbf{a}_{\text {con }} \\
(\mathbf{d e g})\end{array}$ & $\begin{array}{c}\mathbf{O R}_{\max } \\
(\mathbf{m m})\end{array}$ & $\begin{array}{c}\mathbf{C R}_{\max } \\
(\mathbf{m m})\end{array}$ & $\begin{array}{l}a_{1} / a_{2} \\
(d e g)\end{array}$ \\
\hline Romeo 2.5 & \multirow{2}{*}{2.8} & \multirow{2}{*}{0.8} & \multirow{2}{*}{2.1} & \multirow{2}{*}{1.3} & 2.5 & 2.9 & 2.1 & \multirow{2}{*}{$5 / 20$} \\
\hline Romeo 7.0 & & & & & 7.0 & 3.7 & 4.5 & \\
\hline
\end{tabular}

650

651 Table 2: The material properties of three different SRPFs used for the parametric

652 study of under-tapping impact on screw pullout (Sawbones, Worldwide, Pacific

653 Research Laboratories Inc.) [21].

\begin{tabular}{ccccc}
\hline $\begin{array}{c}\text { Density } \\
(\mathrm{g} / \mathrm{cc})\end{array}$ & $\begin{array}{c}\text { Compressive } \\
\text { Strength } \\
(\mathrm{MPa})\end{array}$ & $\begin{array}{c}\text { Compressive } \\
\text { Modulus } \\
(\mathrm{MPa})\end{array}$ & $\begin{array}{c}\text { Shear } \\
\text { Strength } \\
(\mathrm{MPa})\end{array}$ & $\begin{array}{c}\text { Poisson's } \\
\text { ratio }\end{array}$ \\
\cline { 1 - 4 } 0.08 & 0.6 & 16 & 0.59 & \\
\cline { 1 - 3 } 0.16 & 2.2 & 58 & 1.6 & \multirow{2}{*}{0.3} \\
\cline { 1 - 3 } 0.24 & 4.9 & 123 & 2.8 & \\
\hline
\end{tabular}

654

655 Table 3: The pullout force, pullout displacement and stiffness measured for the

656 Romeo 7.0 and the Romeo 2.5 screw. The respective numerical values are given in

657 brackets for comparison.

\begin{tabular}{|c|c|c|c|c|c|c|}
\hline \multirow[t]{2}{*}{ V } & \multicolumn{3}{|c|}{ Romeo 7.0} & \multicolumn{3}{|c|}{ Romeo 2.5} \\
\hline & $\begin{array}{c}\text { Force } \\
(\mathbf{N})\end{array}$ & $\begin{array}{l}\text { Displacement } \\
(\mathrm{mm})\end{array}$ & $\begin{array}{l}\text { Stiffness } \\
(\mathrm{N} / \mathrm{mm})\end{array}$ & $\begin{array}{l}\text { Force } \\
(\mathbf{N})\end{array}$ & $\begin{array}{l}\text { Displacement } \\
(\mathrm{mm})\end{array}$ & $\begin{array}{l}\begin{array}{l}\text { Stiffness } \\
(\mathrm{N} / \mathrm{mm})\end{array} \\
\end{array}$ \\
\hline$a$ & 385 & 0.83 & 543 & 315 & 0.82 & 433 \\
\hline$b$ & 393 & 0.86 & 558 & 324 & 0.67 & 580 \\
\hline$c$ & 379 & 0.85 & 558 & 317 & 0.70 & 536 \\
\hline$d$ & 391 & 0.88 & 538 & 321 & 0.86 & 443 \\
\hline$e$ & 376 & 0.89 & 507 & 312 & 0.75 & 476 \\
\hline Mean & $385(381)$ & $0.86(0.80)$ & $541(582)$ & $318(326)$ & $0.76(0.76)$ & $494(517)$ \\
\hline STDE & V 7 & 0.02 & 21 & 5 & 0.08 & 63 \\
\hline
\end{tabular}


Figure captions:

660

661 Fig. 1: The pedicle screws used for the realization of the pullout tests (up) and the

662 basic geometrical features of the conical pedicle screws used (down).

663

664

Fig. 2: Schematic representation of the experimental set-up.

665

666

Fig. 3: The FE model of a cylindrical screw's hosting material for the case where

667 screw insertion is performed with under-tapping.

668

669

Fig. 4: The two load steps realized in the case of under-tapping (up) or conical screws

670

(down) to assess the impact of pretension to pullout strength: Pretension development

671 (left) and pullout (right).

672

673

674 test was performed using the Romeo 7.0 screw. The section was pressed against a

675 carbon paper to make the conical hole caused by screw pullout easily distinguishable.

676

677

Fig. 6: The experimentally measured and the numerically estimated force vs.

678 displacement curves for cylindrical screw with under-tapping ratio equal to 1.2 (A)

679 and for the conical screws Romeo 2.5 and Romeo 7.0 (B). For each case the

680 experimental curves correspond to the tests which gave the maximum and minimum

681 pullout force.

682

683 
684 Fig. 7: The distribution of the Von Mises equivalent stress $(\mathrm{Pa})$ during different stages

685 of the simulation of pretension generation (left) and pullout (right) for a cylindrical

686 screw that is inserted with under-tapping.

687

688 Fig. 8: The distribution of the Von Mises equivalent stress (Pa) during different stages

689 of the simulation of pretension generation (left) and pullout (right) for a conical screw

690 that is inserted into a cylindrical threaded hole.

691

692 Fig. 9: The numerically calculated pullout force vs. under-tapping ratio for three

693 different densities of synthetic bone.

694

695 Fig. 10: The pullout force calculated for different conical angles for the cases where

696 initial pretension is included or not to the numerical simulation (A) and the \%

697 difference between these two cases (B).

698

699

700

701

702

703

704

705

706

707

708 
Figure 1:

710
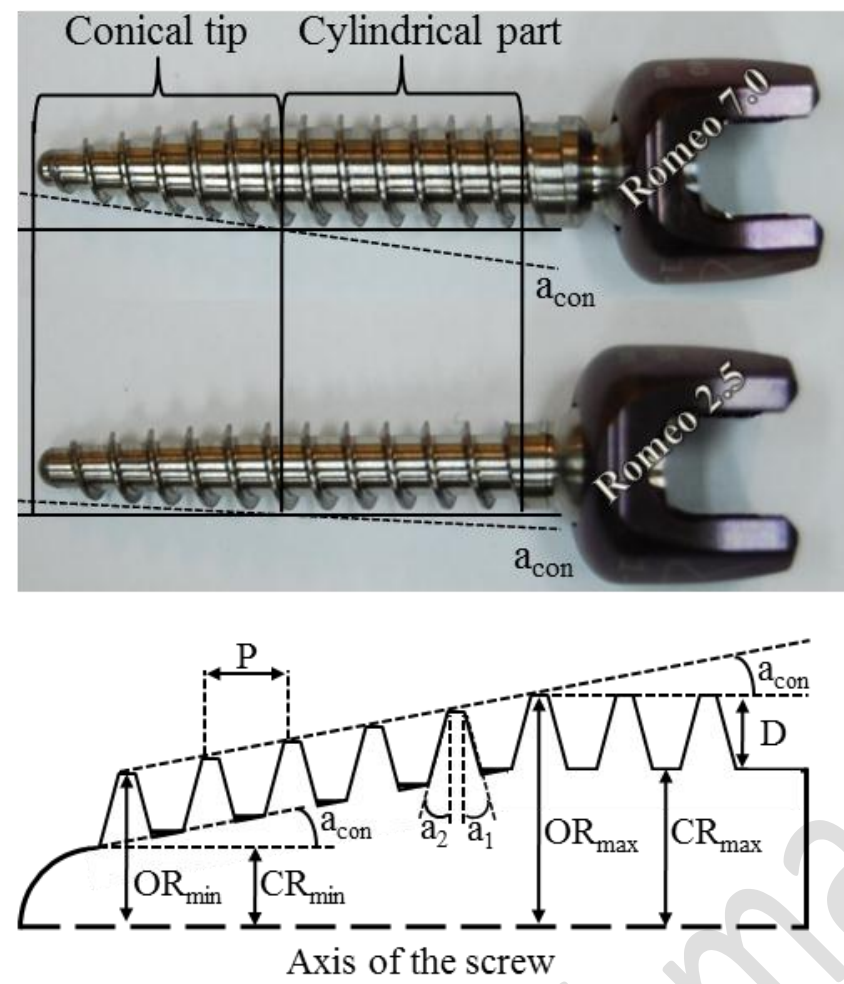

711

712

713

714

715

716

717

718

719

720

721

722

723

724 
725 Figure 2:

726

727

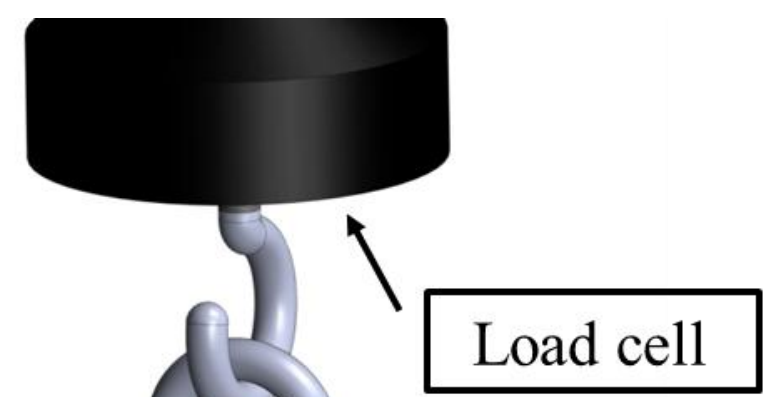

728

729

730

731

732

733

734

735

736

737

738

739 
740 Figure 3:

741

742

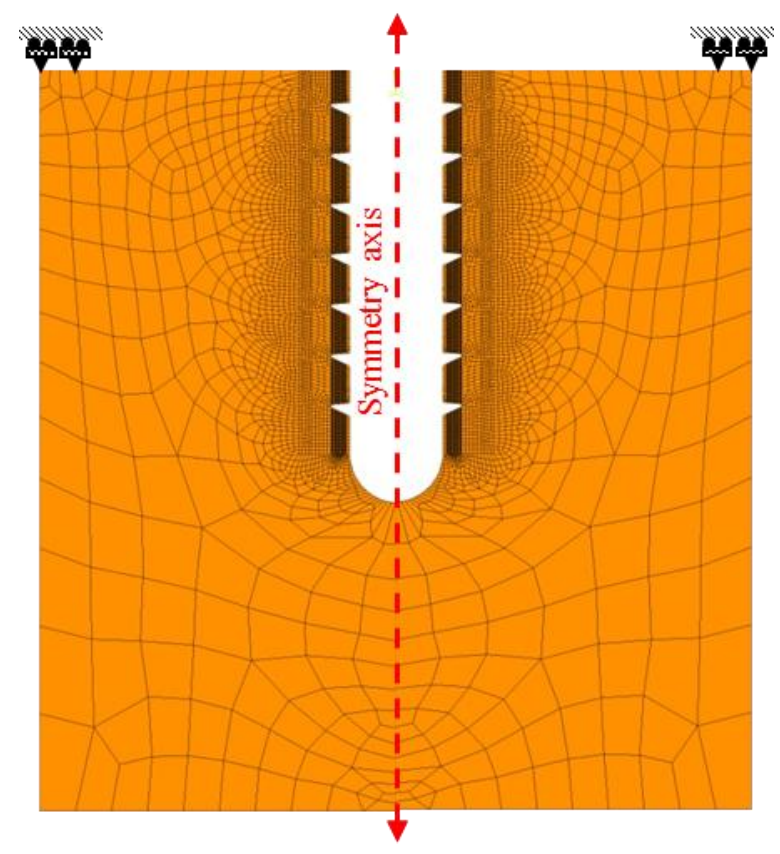

743

744

745

746

747

748

749

750

751

752

753

754

755

756

757 

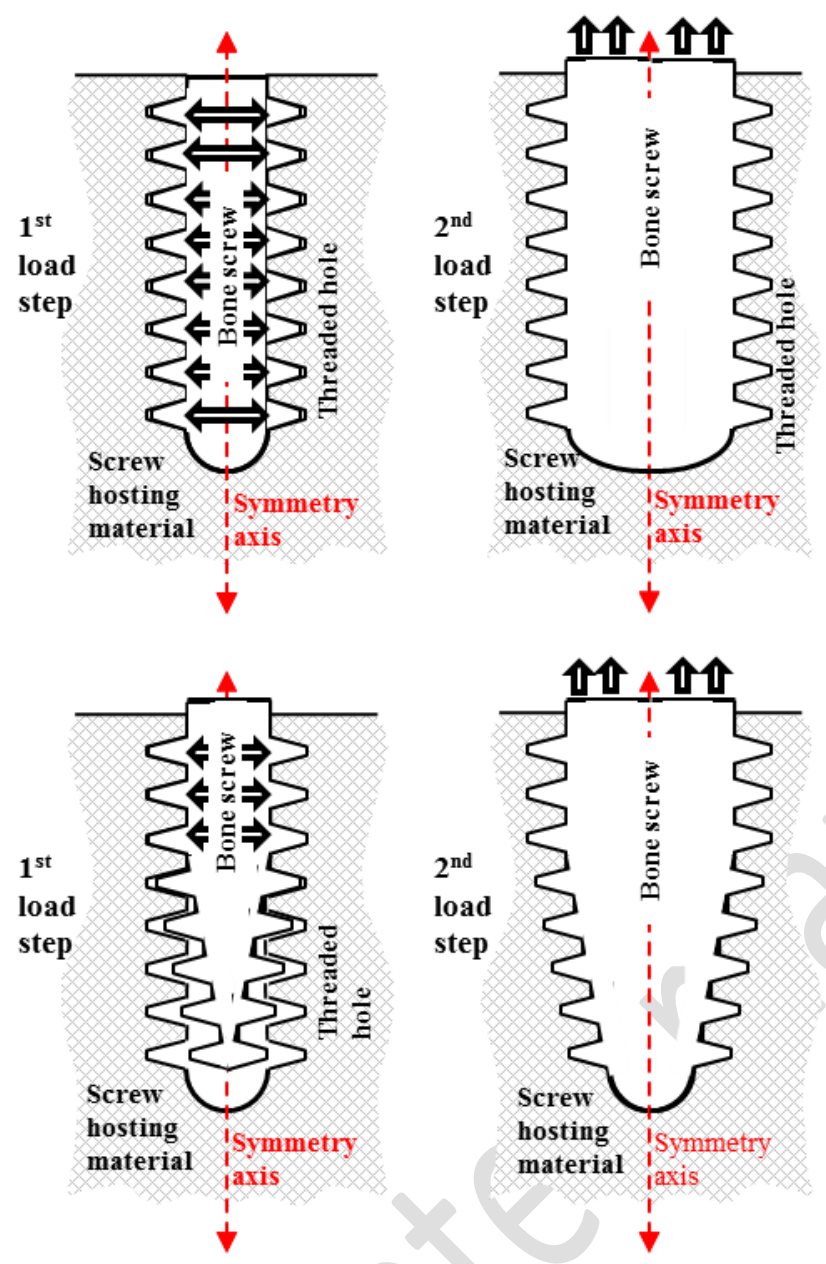
771 Figure 5:

772

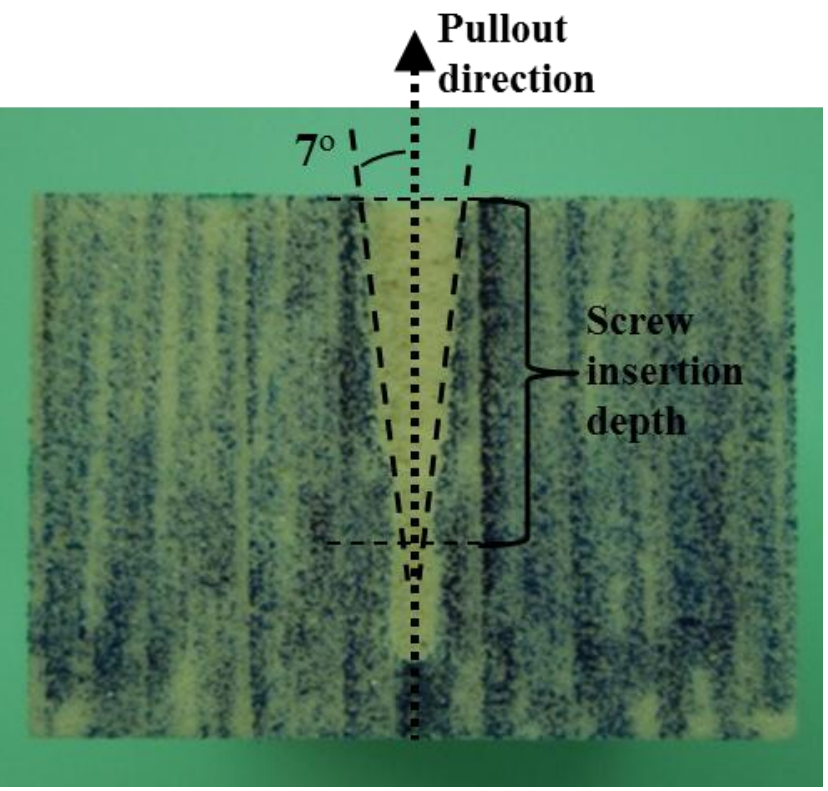

773

774

775

776

777

778

779

780

781

782

783

784

785

786

787

788 
789

Figure 6:

790

791
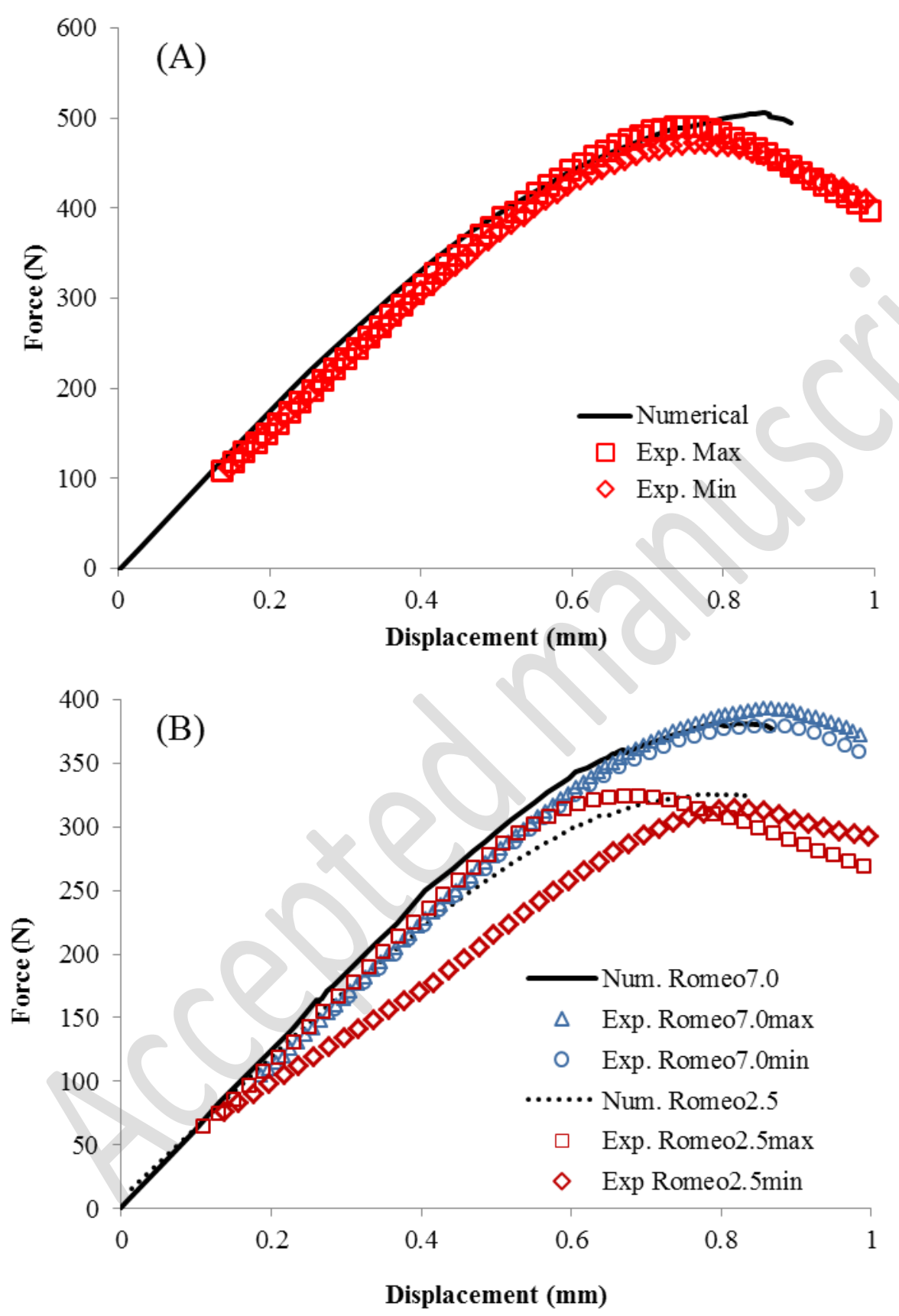

792 
797

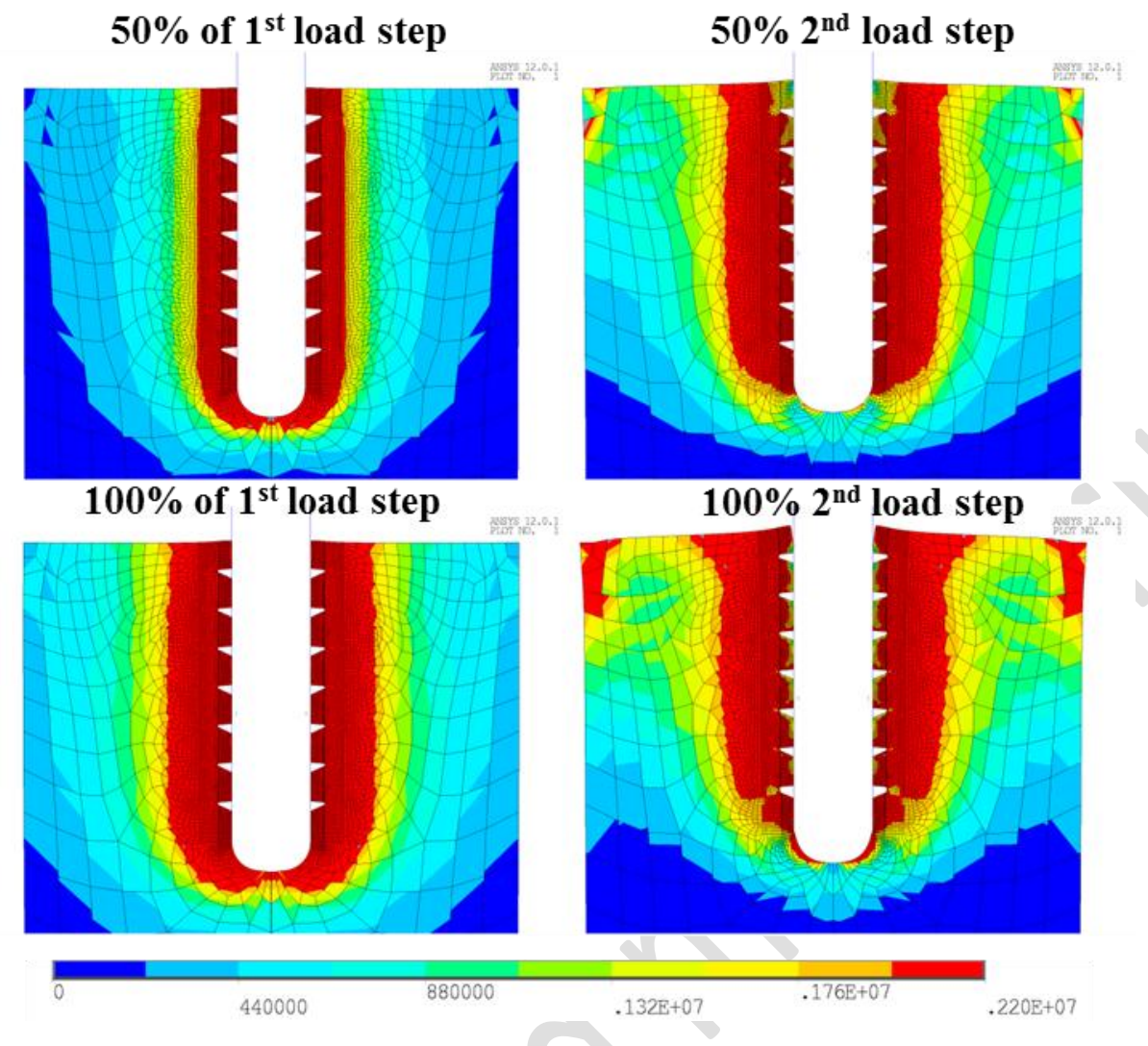

799

800

801

802

803

804

805

806

807

808

809

810 
Figure 8:

812

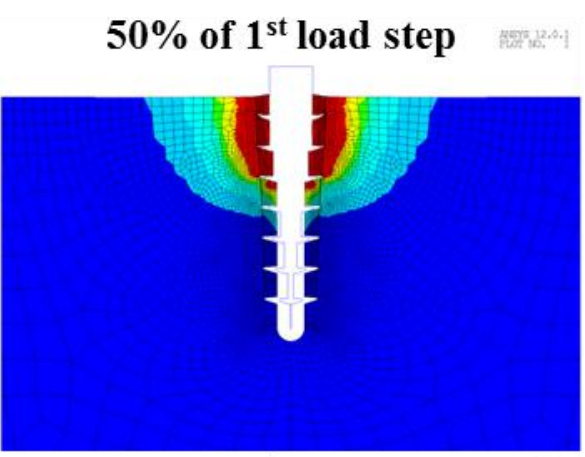

$100 \%$ of $1^{\text {st }}$ load step

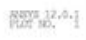
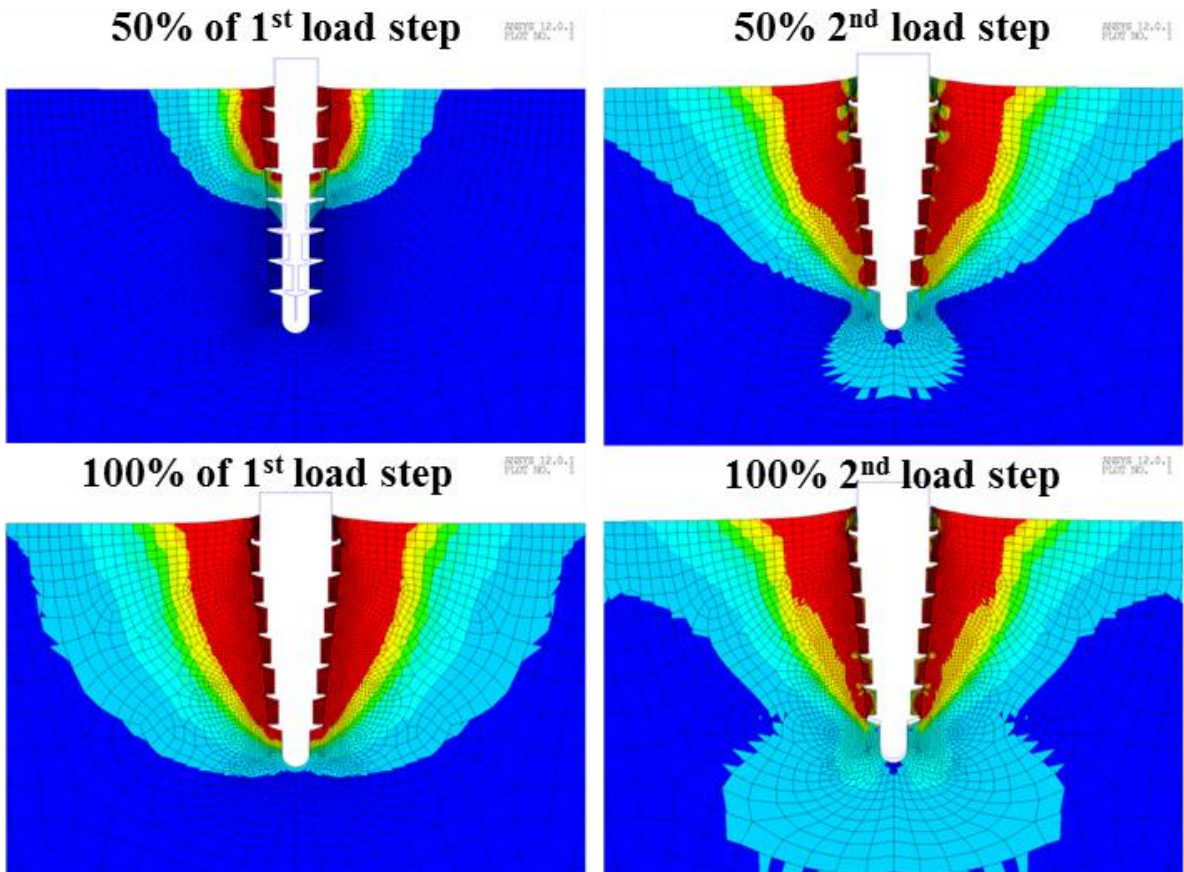

$100 \% 2^{\text {nd }}$ load step

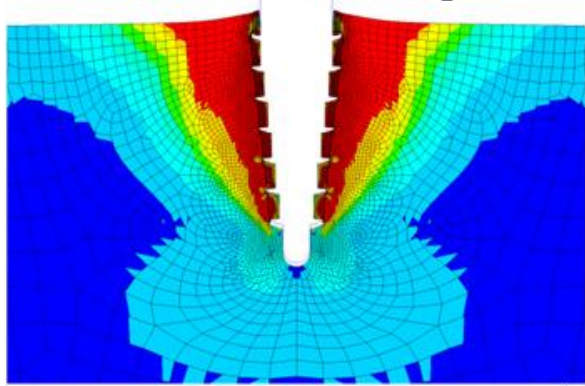

813

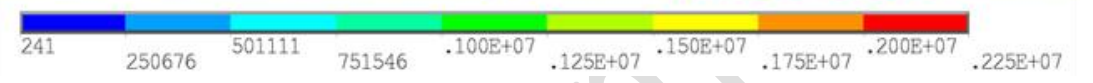

814

815

816

817

818

819

820

821

822

823

824

825

826 
827 Figure 9:

828

829

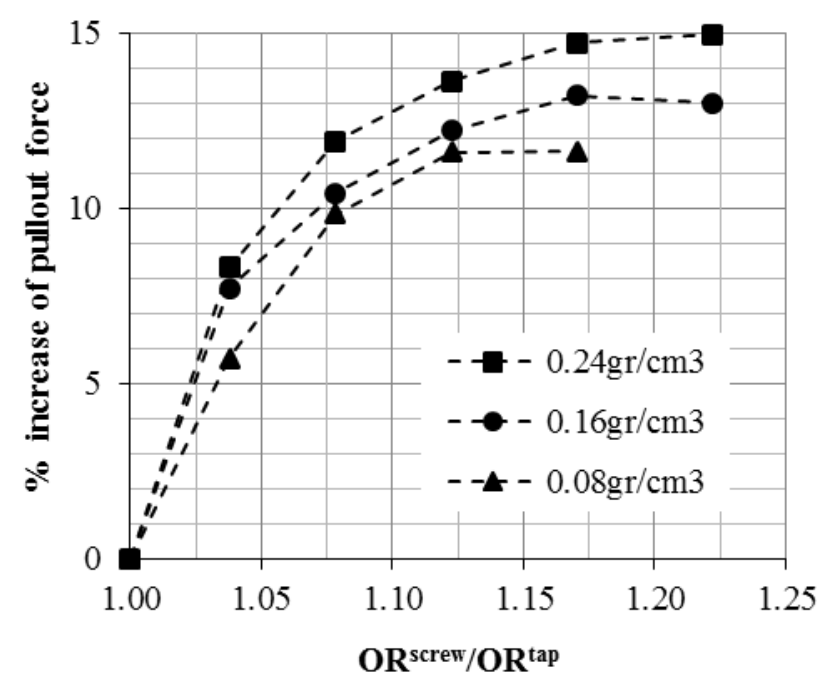

830

831

832

833

834

835

836

837

838

839

840

841

842

843

844

845

846 

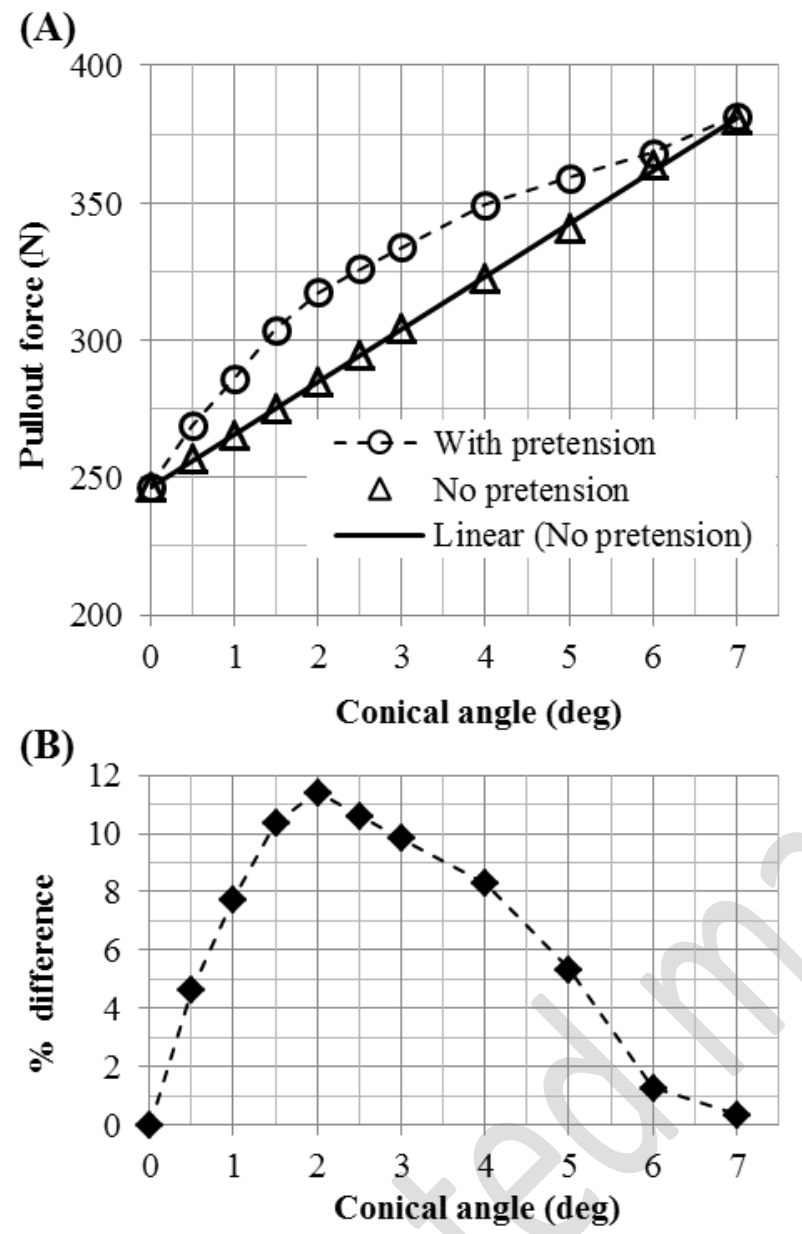

849

850 